\title{
Foreword to the Special Issue on Photocatalysis
}

\author{
Tierui Zhang ${ }^{1} \cdot$ Feng Wang ${ }^{2}$
}

Received: 28 May 2021 / Accepted: 28 May 2021 / Published online: 7 June 2021

(c) Tianjin University and Springer-Verlag GmbH Germany, part of Springer Nature 2021

Massive burning of fossil energy has brought about an energy crisis and also led to a series of environmental problems, such as climate warming and rising sea levels. Photon energy is considered to be an inexhaustible clean energy and is highly valued by researchers. The efficient conversion and utilization of photon energy into heat and electric energy has been widely used in daily production and life, which provides a large amount of clean and renewable highgrade energy for mankind. However, photocatalysis, a direct conversion method of photon energy to chemical energy, is still in the basic research stage due to its low conversion efficiency.

This special issue includes six works (three Reviews and three Research Articles) written by researchers from global top research institutions and universities, including Dalian University of Technology, Wuhan University of Technology, Tianjin University, Chinese Academy of Sciences, Shanghai Normal University and Shenzhen University.

The three original research articles include research areas covering the recent hot topics in photocatalysis focusing on the advanced photocatalytic nanomaterials for water splitting, $\mathrm{CO}_{2}$ reduction and efficient conversion of inert $\mathrm{C}-\mathrm{H}$ bond. More specifically, researchers report three-dimensional hollow octahedral $\mathrm{Cu}_{2} \mathrm{~S} / \mathrm{CdS} \mathrm{p}-\mathrm{n}$ heterostructured architectures for solar-driven water splitting, ultra-thin carbon-doped $\mathrm{Bi}_{2} \mathrm{WO}_{6}$ nanosheets for photocatalytic $\mathrm{CO}_{2}$ reduction and $\mathrm{N}$-doped $\mathrm{TiO}_{2}$ nanotubes for efficient toluene oxidation to benzaldehyde under visible light.

Tierui Zhang

tierui@mail.ipc.ac.cn

1 Key Laboratory of Photochemical Conversion and Optoelectronic Materials, Technical Institute of Physics and Chemistry, Chinese Academy of Sciences, Beijing 100190, China

2 State Key Laboratory of Catalysis, Dalian National Laboratory for Clean Energy, Dalian Institute of Chemical Physics, Chinese Academy of Sciences, Dalian 116023, China
The three reviews closely follow the current research hotspots in the field of photocatalysis, analyze the key issues that have received widespread attention in photocatalysis research, and point out the direction for research in related directions. The researchers summarize the research progress on the photocatalytic synthesis of high-energy-density fuel by using homogeneous and heterogeneous catalytic reaction, mechanism and progress of the photocatalytic oxidation of $\mathrm{NO}_{x}$ and engineering the local coordination environment of single atom catalysts for photocatalytic water splitting.

We are honored to serve as the guest editors of this special issue. We would like to take this opportunity to express our sincere appreciation to the authors for their great support in presenting their excellent research work or reviews for this special issue. This journal will continue to provide opportunities for the publication of high-quality and high-impact papers in the research area of photocatalysis. We sincerely hope that the readers will enjoy the articles published in this special issue.

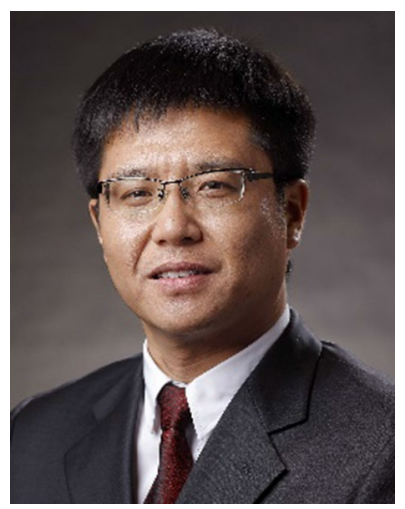

Tierui Zhang is a full professor at the Technical Institute of Physics and Chemistry, Chinese Academy of Sciences. He obtained his Ph.D. degree in Chemistry in 2003 at Jilin University (China). Subsequently, he worked as a postdoctoral researcher in the laboratories of Prof. Markus Antonietti, Prof. Charl F. J. Faul, Prof. Hicham Fenniri, Prof. Z. Ryan Tian, Prof. Yadong Yin, and Prof. Yushan Yan. His current scientific interests focus on the catalytic nanomaterials for energy conversion. 